\section{Melatonin zaps $\mathrm{T}_{\mathrm{H}} 17$}

Pathogenic responses of the $T_{H} 17$ subset of helper $T$ cells are associated with disease flares in patients with multiple sclerosis, who also show unexplained seasonal variation in relapses. In Cell, Farez et al. report that melantonin interferes with expression of the transcription factor ROR $\gamma \mathrm{t}$, a key driver of the polarization and effector function of $T_{H} 17$ cells. The concentration of melatonin in serum is inversely correlated with the abundance of $T_{H} 17$ cells and multiple sclerosis flares, which suggests a possible role for melatonin in this seasonal variation. Melatonin signaling activates an Erk kinase-expression pathway that relieves inhibition of expression of the transcription factor NFIL3, which itself inhibits expression of the gene encoding ROR $\mathrm{t}$. Mice lacking expression of melatonin receptors fail to repress $\mathrm{T}_{\mathrm{H}} 17$ differentiation. Melatonin also enhances interleukin 10 expression to induce type 1 regulatory T cells. Thus, seasonal variation in melatonin expression alters the balance between these cell populations and thereby alters the pathogenic immune responses associated with multiple sclerosis flares. $L A D$ Cell 162, 1338-1352 (2015)

\section{Preserving intestinal stem cells}

In the intestine, crypt-residing stem cells proliferate continuously to replenish the epithelial barrier. Because of this high mitotic activity, the gut epithelium is often affected by anticancer therapies. In The Journal of Experimental Medicine, Cupedo and colleagues use a model of methotrexate-induced self-resolving damage in the small intestine to show that type 3 innate lymphoid cells (ILC3 cells) are required for the proliferation and regeneration of epithelial cells. After treatment with methotrexate, both NKp $46^{+}$ILC3 cells and CCR6 ${ }^{+}$ILC3 cells are activated and express the cytokines IL-22, TNF, LT $\beta$ and IFN- $\gamma$, and ILC3-deficient ROR $\gamma$ t-deficient mice have more damage in the intestinal crypt and loss of Lgr $5^{+}$intestinal stem cells than do wild-type mice. Blockade of IL-22 also leads to loss of intestinal stem-cell maintenance in the duodenum. The role of ILC3 in preserving intestinal stem cells could be harnessed to minimize side effects in patients undergoing cytotoxic therapy.

J. Exp. Med. (21 September 2015) doi:10.1084/jem.20150318

\section{Don't eat me, activate me}

The signal-regulatory protein CD47 mediates a "don't eat me" signal when it binds its receptor SIRPa, and this is thought to contribute to the resistance of tumors to phagocyte-dependent clearance. In Nature Medicine, $\mathrm{Xu}$ and colleagues show that therapeutic blockade of CD47 also leads to the activation of potent anti-tumor cytotoxic T lymphocyte (CTL) responses. Using syngeneic mouse tumor models, the authors find that blocking CD47 allows the sensing of tumor DNA and then triggers type I interferons. Acting on tumorinfiltrating dendritic cells, the type I interferons then drive the crosspresentation of tumor antigens to CTLs. These CTLs are essential for the anti-tumor effects of CD47 blockade. Therefore, in addition to simply preventing the phagocytosis of tumor cells, the CD47-SIRPa axis also protects tumors from destructive CTL responses. These findings reveal a new perspective on the underlying mechanism of therapeutic CD47 blockade.

Nat. Med. (31 August 2015) doi:10.1038/nm.3931

\section{cGAS ramps up autoinflammatory disease}

The cystosolic DNA sensor cGAS catalyzes production of the second messenger cGAMP. In the Journal of Immunology, a paper by Stetson and colleagues, and in the Proceedings of the National Academy of Sciences USA, a separate study by Chen and colleagues, show that cGAS can sense aberrantly accumulated self DNA and initiate autoinflammatory disease. Deficiency in cellular exonucleases or endonucleases leads to the accumulation of cytosolic DNA, which then triggers excessive type I interferon via DNA sensors and autoinflammatory disease. However, nuclease-deficient mice that also lack cGAS are 'rescued' from a rampant type I interferon signature and disease. Even haploinsufficiency in cGAS can partially protect mice from the absence of certain nucleases. cGAMP is sensed downstream by the adaptor STING, whose knockout is similarly protective. Mice deficient in both the nuclease TREX1 and STING still accumulate cGAMP, but this is not pathogenic. Thus, cGAS is important for the recognition of cytosolic DNA and in driving certain autoinflammatory diseases. ZF Proc. Natl. Acad. Sci. USA 112, 12903-12904 (2015) \& J. Immunol. 195, 1939-1943 (2015)

\section{Aging neutrophils}

Aged neutrophils are known to upregulate the chemokine receptor CXCR4, which might allow their clearance from the bone marrow, and downregulate L-selectin (CD62L). In Nature, Frenette and colleagues show that aged circulating $\mathrm{CD} 62 \mathrm{~L}^{\mathrm{lo}} \mathrm{CXCR} 4^{\text {hi }}$ neutrophils in mice have more activation of integrin $\alpha_{M} \beta_{2}$ (Mac-1) and greater ROS production and form neutrophil extracellular traps. Aged neutrophils are distinct from activated neutrophils but upregulate several activation pathways, including adhesion pathways and signaling via TLRs, NLRs and NF-kB. Microbiota-derived signals induce neutrophil aging through TLR2-, TLR4- and MyD88mediated pathways but not TNF- or GM-CSF-mediated pathways. Mouse models and patients with sickle cell disease have a greater number of aged neutrophils, and antibiotic treatment lowers the total number of neutrophils and the frequency of aged neutrophils in both mice and humans.

\title{
SLE links to Alu elements
}

Patients with systemic lupus erythematosus have autoantibodies that recognize RNA-binding proteins and have type I interferon signatures. In Science, Behrens and colleagues show that Ro60, a known lupus autoantigen, forms complexes with RNAs expressed from Alu element-expressing endogenous retroviruses. Patients with lupus have higher concentrations of Ro60-Alu-containing RNA in their serum than do healthy control subjects. Ro60 recognizes a 3' untranslated region in Alu RNA. Knockdown of Ro60 in human B cell lines increases the abundance of Alu RNA, which suggests that Ro60 acts to limit the availability of Alu RNA to endogenous RNA sensors. A feed-forward inflammatory loop seems to exist, as type I interferon drives Alu expression, which in turn activates TLR7 signaling to increase interferon expression. The authors speculate that this Alu-recognition pathway served as an evolutionary advantage to enhance inflammatory antiviral responses but results in heightened autoimmunity.

$L A D$

Science (17 September 2015) doi:10.1126/science.aac7442 


\section{Erratum: cGAS ramps up autoinflammatory disease}

\section{Zoltan Fehervari}

Nat. Immunol. 16, 1113 (2015); published 20 October 2015; corrected after print 7 January 2016

In the version of this Research Highlight initially published, only the work of Gao et al. (Proc. Natl. Acad. Sci. USA 112, 12903-12904 (2015)) was discussed. This has been revised to include discussion of another article with very similar data published a few weeks earlier, by Gray et al. (J. Immunol. 195, 1939-1943 (2015)). The error has been corrected in the HTML and PDF versions of the article. 